\title{
Research Trends of Systematic Review/Meta-Analysis on Acupuncture Therapy: A Bibliometric Analysis
}

This article was published in the following Dove Press journal:

Journal of Pain Research

\author{
Jinlan Jiang $\mathbb{D}^{1, *}$ \\ Jiaxin Zhang $\mathbb{D D}^{2, *}$ \\ Rongrong $\mathrm{Li}^{\prime}$ \\ Zhengqi Zhao ${ }^{2}$ \\ Xiaolin $\mathrm{Ye} \mathbb{D}^{2,3}$
}

'The Third Clinical Medical College, Zhejiang Chinese Medical University, Hangzhou City, Zhejiang Province, People's Republic of China; ${ }^{2}$ College of Basic Medical Science, Zhejiang Chinese Medical University, Hangzhou City, Zhejiang Province, People's Republic of China; ${ }^{3}$ The Second Affiliated Hospital of Zhejiang Chinese Medical University, Hangzhou City, Zhejiang Province,

People's Republic of China

*These authors contributed equally to this work
Correspondence: Xiaolin Ye

College of Basic Medical Science, Zhejiang Chinese Medical University, Hangzhou

City, Zhejiang Province, People's Republic of China

Tel $+86 \quad$ I8858I42475

Email yex184@aliyun.com
Objective: We aimed to explore the hotspots and frontiers of systematic review/metaanalysis on acupuncture therapy according to bibliometric methods.

Methods: Systematic review/meta-analysis articles on acupuncture therapy were retrieved from the Web of Science Core Collection. We used CiteSpace 5.6.R5 to analyze annual publication, categories, countries, institutions, journals, authors, cited references, and keywords through visual maps to explore the research hotspots and trends.

Results: An analysis of 1189 articles in English showed that the total number of publications continually increased in the last 12 years. General medicine was the most used category, and the country and institution ranking highest in this field were China and Beijing University of Chinese Medicine, respectively. The Journal of Alternative and Complementary Medicine was the most prolific journal, and Cochrane Database of Systematic Reviews was the most cited journal. Myeong Soo Lee was the most productive author, and J.P.T. Higgins ranked first in frequency among the cited authors. In the cited references, the top two were Cochrane Handbook for Systematic Reviews of Interventions and the Standards for Reporting Interventions in Clinical Trials of Acupuncture (STRICTA). As for keywords, there were 6 main frontiers including interventions, disease, patient, comparisons, study types, and outcomes. The first disease was pain, while the intervention was electroacupuncture (EA).

Conclusion: The present study examined the research trends in systematic reviews and meta-analysis on acupuncture therapy through bibliometric methods and revealed potential future hotspots of research in this field.

Keywords: acupuncture, systematic review, meta-analysis, CiteSpace, bibliometric analysis

\section{Introduction}

Evidence-based medicine (EBM) refers to the medical process that applies the best research evidence to clinical practice. ${ }^{1}$ The evidence is mainly from randomized controlled trials with large samples and systematic review/meta-analysis. ${ }^{2}$ As the core research method of EBM, meta-analysis collects quantities of current research results and conducts a comprehensive and systematic quality evaluation to reach reliable conclusions, ${ }^{3}$ which is at the top of the evidence pyramid with high repeatability and reliability. ${ }^{4,5}$

It is necessary to introduce EBM into the field of traditional Chinese medicine (TCM) characterized by syndrome differentiation and treatment if we want to make TCM from individual to group. ${ }^{6,7}$ Acupuncture, as one of the important disciplines in TCM, is a simple, efficient, economical, and safe means for treating pain syndromes, ${ }^{8-11}$ chronic 
inflammatory disorders, ${ }^{12,13}$ and other indications and has become a major part of international complementary and alternative medicine. ${ }^{14}$ With the development of EBM, the quantity of literature on systematic review/meta-analysis and guidelines related to acupuncture therapy is increasing, which means that acupuncture is becoming a standardized and normal therapy. However, there is still a lack of summary and evaluation of the literature and research results on acupuncture, as well as the classification of research directions, promising and popular research areas, and developmental trends in this field.

CiteSpace is visualization software that can identify new trends and demonstrate new dynamics. ${ }^{15,16}$ Through co-occurrence and co-citation analysis of authors, keywords, and references in related literature, we used CiteSpace to explore the current status of research on systematic review/meta-analysis of acupuncture therapy.

\section{Materials and Methods}

\section{Data Collection}

All data were obtained from the Web of Science Core Collection via the Zhejiang Chinese Medical University Libraries website on August 30th, 2020. It included only those articles and reviews in English without time limitations. The strategy consisted of three parts. First, "acupuncture" was used as the subject term, combined with corresponding free terms such as electroacupuncture, auricular acupuncture, moxibustion, etc., and a total of 13,152 records were retrieved. We then used "meta-analysis" and "systematic review" as the subject terms, combined with corresponding free terms such as meta-analysis, which retrieved a total of 265,143 records. Finally, we merged the above two queries, and 1786 records were retrieved (See Table 1 for specific search strategies and results).

\section{Analysis Tools}

We manually deleted duplicate and irrelevant data. As a result, we retained 1189 records. Then, we imported the data into CiteSpace5.6.R5 to perform bibliometric analysis. The visual map mainly included nodes and the links between nodes. The size of the nodes indicated frequency, and the links between nodes meant that these two nodes appeared together in the same literature. The thicker the links were, the more co-occurrence they had. In addition, the color of links represented the year they first appeared. The color of the nodes represented the year as well, and the warmer the color, the newer the year. ${ }^{17}$ The node with a purple ring showed high centrality, connecting the other two nodes and
Table I The Topic Search Queries

\begin{tabular}{|l|l|l|}
\hline Set & Results & Search Query \\
\hline$\# I$ & 13,152 & $\begin{array}{l}\text { TOPIC: (acupuncture) OR TOPIC: (acupuncture } \\
\text { therapy) OR TOPIC: (Acupuncture Points) OR } \\
\text { TOPIC: (acupuncture point) OR TOPIC: } \\
\text { (Acupoint) OR TOPIC: (Acupoints) OR TOPIC: } \\
\text { (Acupuncture, Ear) OR TOPIC: (Auricular } \\
\text { Acupuncture) OR TOPIC: (body acupuncture) OR } \\
\text { TOPIC: (Electroacupuncture) OR TOPIC: (electro- } \\
\text { acupuncture) OR TOPIC: (Moxibustion) AND } \\
\text { DOCUMENT TYPES: (Article OR Review) AND } \\
\text { LANGUAGE: (English) Indexes=SCI-EXPANDED, } \\
\text { CCR-EXPANDED, IC Timespan=All years }\end{array}$ \\
\hline$\# 2$ & 265,143 & $\begin{array}{l}\text { TOPIC: (Network Meta-Analysis) OR TOPIC: } \\
\text { (Meta-Analysis) OR TOPIC: (meta analysis) OR } \\
\text { TOPIC: (Systematic Review) AND DOCUMENT } \\
\text { TYPES: (Article OR Review) AND LANGUAGE: } \\
\text { (English) Indexes=SCI-EXPANDED, CCR- } \\
\text { EXPANDED, IC Timespan=All years }\end{array}$ \\
\hline$\# 3$ & I786 & \#I AND \#2 \\
\hline
\end{tabular}

playing an important intermediary role and is usually perceived as representing a turning point in a field. ${ }^{18}$ The parameters of CiteSpace5.6.R5 were set as below: (1)Time slicing was from 2008 to 2020, years per slice. ${ }^{1}$ (2)All options in the term source were selected. (3)one node type was selected at a time. (4)All thresholds were (c,c,ccv:2,2,20;4,3,20;4,3,20) except Figure 4B (c,c,ccv:10,10,20;4,3,20;4,3,20) and Figure 5A-C (c,c,ccv:5,5,20;4,3,20;4,3,20). (5)Each figure's pruning was (pathfinder, pruning sliced networks and pruning the merged network) except Figures $4 \mathrm{~A}$ and $5 \mathrm{~A}-\mathrm{C}$ (without pruning).

\section{Results and Discussion Annual Publication}

A total of 1189 records were included, and the amount of literature had consistently increased over the past 12 years (Figure 1), especially in 2019. It would continue to increase in 2020. From the prominent increase in the amount of literature, we found great importance was attached to EBM in acupuncture, which has also actively utilized modern medical research methods to improve the quality of scientific research and promote the development of acupuncture science.

\section{Analysis of Categories}

After an analysis by CiteSpace, 46 categories were found. Table 2 shows the frequency of the top 10 categories. As 


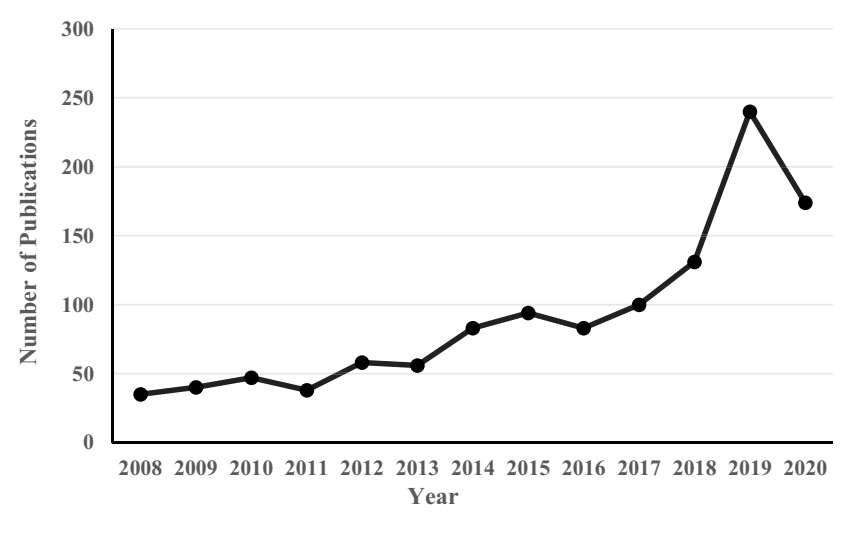

Figure I The annual number of publications on acupuncture therapy related systematic review/meta analysis between 2008 to 2020 .

we can see, Integrative \& Complementary Medicine ranked first with 449 articles, followed by General \& Internal Medicine (340), Neuroscience \& Neurology (102), Clinical Neurology (82), Obstetrics \& Gynecology (47). Oncology (32), in ninth place, ranked first in centrality (0.36). Through categories analysis, we learned that systematic review and meta-analysis related to acupuncture had covered most medical disciplines, especially general medicine, neurology, obstetrics and gynecology, and oncology.

\section{Analysis of Journals and Cited Journals}

The top 10 journals are listed by the number of publications in Table 3. In general, they were mainly comprehensive such as the Journal of Alternative and Complementary Medicine

Table 2 The Top 10 Categories on Acupuncture Therapy Related Systematic Review/Meta Analysis

\begin{tabular}{|l|l|c|}
\hline Rank & Freq & Categories \\
\hline 1 & 449 & Integrative \& Complementary Medicine \\
\hline 2 & 340 & General \& Internal Medicine \\
\hline 3 & 102 & Neuroscience \& Neurology \\
\hline 4 & 82 & Clinical Neurology \\
\hline 5 & 47 & Obstetrics \& Gynecology \\
\hline 6 & 40 & Science \& Technology - other topics \\
\hline 7 & 40 & Multidisciplinary Sciences \\
\hline 8 & 40 & Neurosciences \\
\hline 9 & 32 & Oncology \\
\hline 10 & 26 & Research \& Experimental Medicine \\
\hline
\end{tabular}

Table 3 The Top 10 Journals on Acupuncture Therapy Related Systematic Review/Meta Analysis

\begin{tabular}{|l|c|c|c|}
\hline Rank & Publications & Journal & $\begin{array}{c}\text { IF } \\
\mathbf{( 2 0 1 9 )}\end{array}$ \\
\hline 1 & 13 & $\begin{array}{c}\text { Journal of Alternative and } \\
\text { Complementary Medicine }\end{array}$ & 2.109 \\
\hline 2 & 12 & $\begin{array}{c}\text { Complementary Therapies in } \\
\text { Medicine }\end{array}$ & 2.063 \\
\hline 3 & 11 & $\begin{array}{c}\text { Evidence-based Complementary } \\
\text { and Alternative Medicine }\end{array}$ & 1.813 \\
\hline 4 & 11 & $\begin{array}{c}\text { European Journal of Integrative } \\
\text { Medicine }\end{array}$ & 0.974 \\
\hline 5 & 11 & $\begin{array}{c}\text { BMC Complementary and } \\
\text { Alternative Medicine }\end{array}$ & 2.833 \\
\hline 6 & 9 & PLoS One & 2.74 \\
\hline 7 & 8 & $\begin{array}{r}\text { Chinese Journal of Integrative } \\
\text { Medicine }\end{array}$ & 1.545 \\
\hline 10 & 7 & Supportive Care in Cancer & 2.635 \\
\hline 8 & 8 & Neural Regeneration Research & 3.171 \\
\hline
\end{tabular}

(J Altern Complem Med) without significant difference in the number of publications from Neural Regeneration Research (Neural Regen Res) and Supportive Care in Cancer (Support Care Cancer) and it suggested that acupuncture had been widely used in the treatment of tumor and neurological diseases to some extent.

A cited journal map (Figure 2) included 680 nodes and 951 links, and top 5 among them in frequency and centrality are shown in Table 4. The cited journals could be divided into four types: the first was associated with acupuncture, with a relatively low impact factor, such as Acupuncture in Medicine (Acupunct Med), Journal of Alternative and Complementary Medicine (J Altern Complem Med), Evidence-Based Complementary and Alternative Medicine (Evid-based Compl Alt), and Clinical Journal of Acupuncture and Moxibustion (J Clin Acupunct Moxi). The second was related to disease, such as Anesthesiology (Anesthesiology), Sleep Medicine Reviews (Sleep Med Rev), Support Care in Cancer (Support Care Cancer). Cochrane Database of Systematic Reviews (Cochrane Db Syst Rev) was the third type in connection with EBM, and BMJ-British Medical Journal (BMJ-Brit Med J), Hong Kong Medical Journal (Hong Kong Med J) vesting in synthesizing journal 


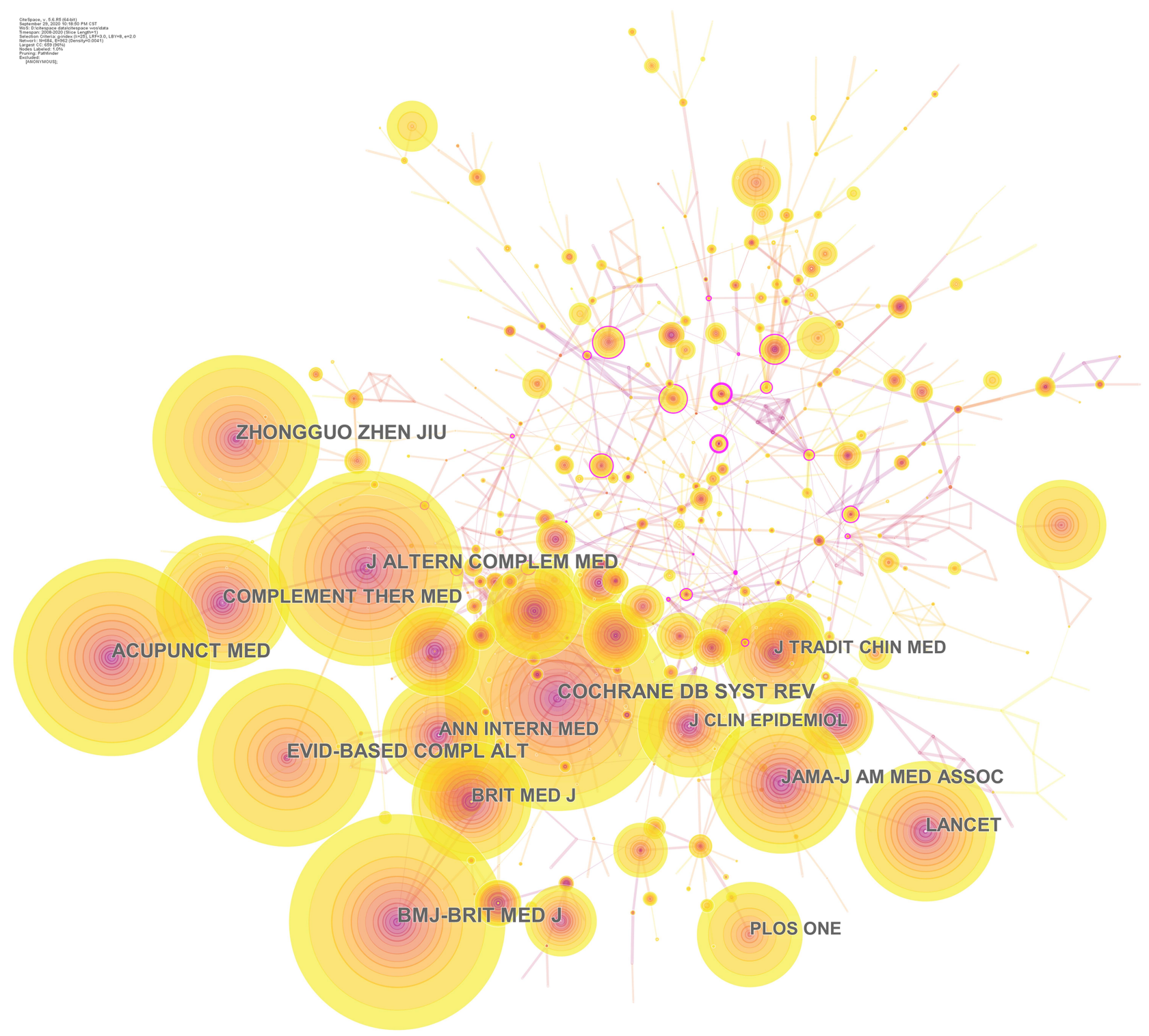

Figure 2 The cited journal map on acupuncture therapy related systematic review/meta analysis. (The nodes in the map represent journals, and lines between the nodes represent co-citation relationships. The various colors in the nodes represent the different years. The larger the node area, the greater the number of citations. The purple ring represents centrality, and nodes with high centrality are considered as pivotal points in the literature.).

were the last type. BMJ-Brit Med J with a high impact factor and ranked second in the frequency of cited journals. In addition, two of its documents about PRISMA statements for systematic review and meta-analysis protocols and the Cochrane Collaboration tool for randomized trials also ranked in the frequency of cited references. As a higher-level evidence, RCT is often used for meta-analysis in order to obtain credible results.

\section{Distribution of Countries and Institutions}

The distribution of countries map (Figure 3A) shows 45 countries had published related literature, and the top five countries are displayed in Table 5. China was the most productive one, publishing 683 articles, surpassing South Korea (196), England (145), United States (138), and Australia (91). In terms of centrality, the top five countries were Italy (1.12), Sweden (0.8), Denmark (0.55), Switzerland (0.41), and Germany (0.39), so we could see Italy and Sweden had close cooperation with other countries in the systematic review and meta-analysis of acupuncture. In addition, some countries formed small-scale cooperative groups, but there was a lack of cooperation among countries. However, it did not fully represent the lack of cooperation in the application of acupuncture therapy among them. 
Table 4 The Top 5 Frequency and Centrality of Cited Journals on Acupuncture Therapy Related Systematic Review/Meta Analysis

\begin{tabular}{|c|c|c|c|c|c|c|c|}
\hline Rank & Freq & Cited Journal & $\begin{array}{c}\text { IF } \\
(2019)\end{array}$ & Rank & Centrality & Cited Journal & $\begin{array}{c}\text { IF } \\
(2019)\end{array}$ \\
\hline 1 & 610 & Cochrane Database of Systematic Reviews & 30.223 & I & 0.27 & Anesthesiology & 7.067 \\
\hline 2 & 588 & BMJ-British Medical Journal & 7.89 & 2 & 0.24 & $\begin{array}{c}\text { Journal of Clinical Acupuncture and } \\
\text { Moxibustion }\end{array}$ & l \\
\hline 3 & 537 & Acupuncture in Medicine & 2.219 & 3 & 0.17 & Sleep medicine Reviews & 9.613 \\
\hline 4 & 532 & $\begin{array}{l}\text { Journal of Alternative and Complementary } \\
\text { Medicine }\end{array}$ & 2.109 & 4 & 0.15 & Supportive Care in Cancer & 2.635 \\
\hline 5 & 493 & $\begin{array}{c}\text { Evidence-based Complementary and } \\
\text { Alternative Medicine }\end{array}$ & 1.813 & 5 & 0.15 & Hong Kong Medical Journal & 1.679 \\
\hline
\end{tabular}

The distribution of institutions map (Figure 3B) consists of 425 nodes and 627 links. Table 6 shows the top five institutions and Beijing University of Chinese Medicine published the most articles (113), followed by KyungHee University (102), which also ranked in the top three in terms of centrality. Generally speaking, the institutions were mostly universities, and some were hospitals. Chengdu University of Traditional Chinese Medicine, as one of the EBM centers, published 87 articles, and its affiliated hospital (Hospital Chengdu University of Traditional Chinese Medicine) also published 19 articles. However, another EBM center, Sichuan University, had fewer than 20 articles. Cooperation among institutions was mostly national, except for some transnational cooperation, for example, RMIT University located in Australia cooperated with Guangzhou University of Chinese

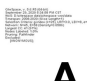

A
B
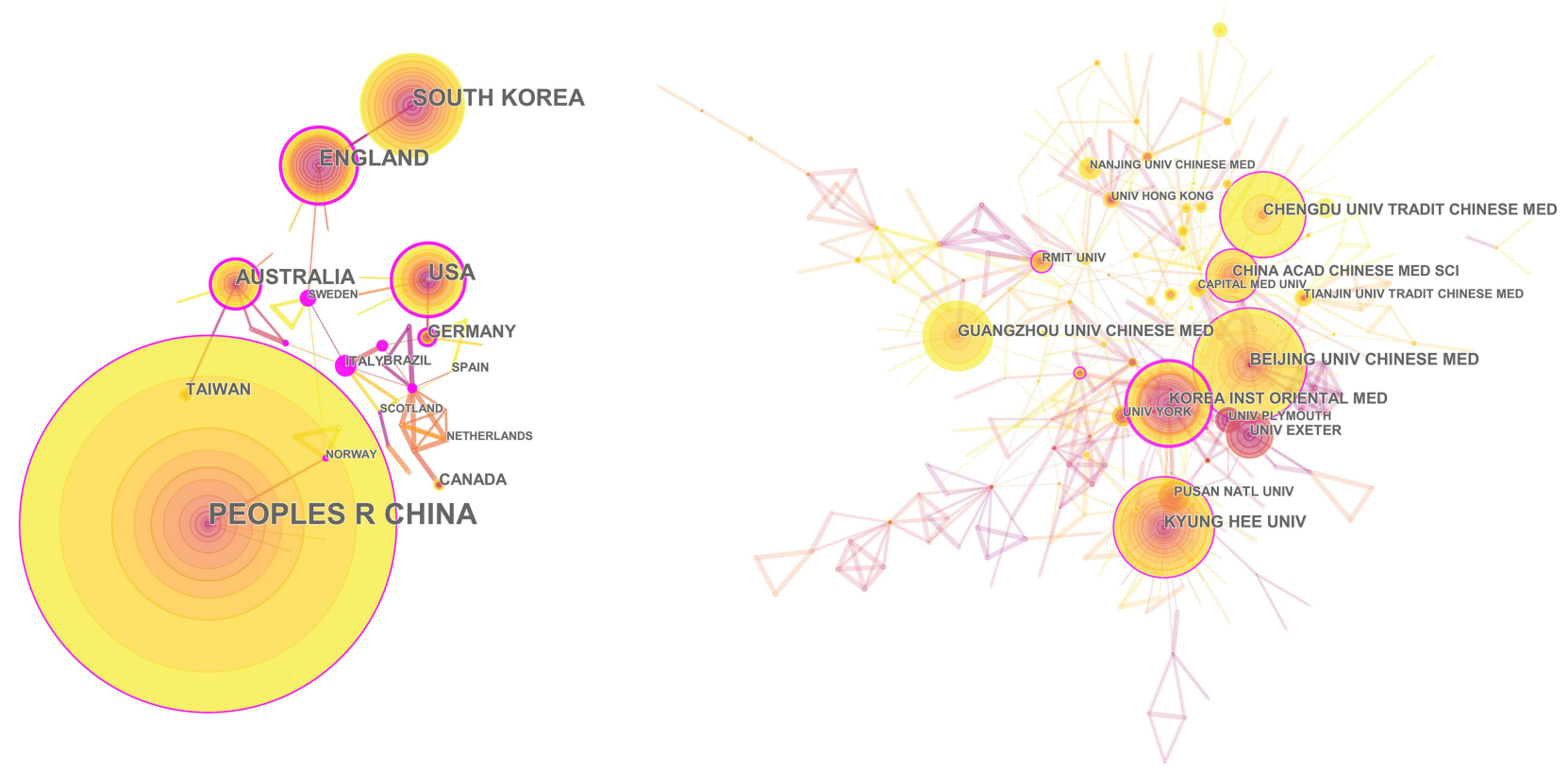

Figure 3 (A) shows the countries map on acupuncture therapy related systematic review/meta analysis (The nodes in the map represent countries. The lines between the nodes represent cooperation relationships. The various colors in the nodes represent the different years, and the larger the area of the node, the larger the number of publications. The purple ring represents centrality, and nodes with high centrality are considered as pivotal points in the literature.). (B) shows the institutions map on acupuncture therapy related systematic review/meta analysis (The nodes in the map represent institutions. The lines between the nodes represent cooperation relationships. The various colors in the nodes represent the different years, and the larger the area of the node, the larger the number of publications. The purple ring represents centrality, and nodes with high centrality are considered as pivotal points in the literature.). 
A

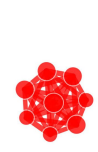

\section{B}

B
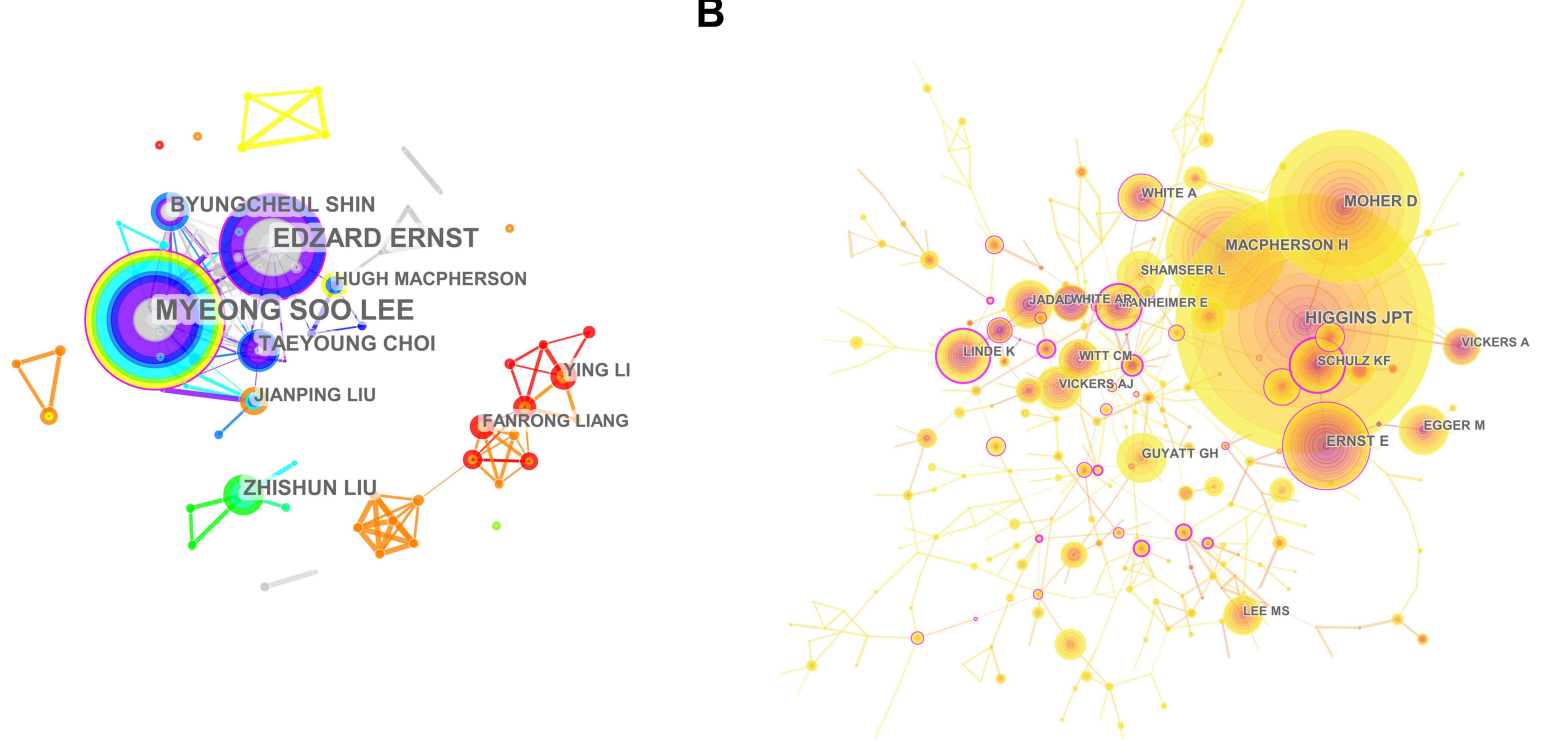

Figure 4 (A) shows the authors map on acupuncture therapy related systematic review/meta analysis (The nodes in the map represent authors, and lines between the nodes represent the collaborative relationships. The various colors in the nodes represent the different years, and the larger the node area, the greater the number of publications.). (B) shows the cited authors map on acupuncture therapy related systematic review/meta analysis (The nodes in the map represent cited authors, and lines between the nodes represent co-citation relationships. The various colors in the nodes represent the different years. The larger the node area, the greater the number of citations. The purple ring represents centrality, and nodes with high centrality are considered to be pivotal points in the literature.).

Medicine and Nanjing University of Chinese Medicine, and the Korea Institute of Oriental Medicine collaborated with the University of Exeter of England and Beijing University of Chinese Medicine. Most institutions, however, did not work closely together.

\section{Analysis of Authors and Cited Authors}

The authors co-occurrence map (Figure 4A) shows the cooperative relationships among the authors, and it contains 90 nodes and 198 links. Table 7 shows the top 10 authors in terms of publication volume and centrality. The most prolific author, with 65 papers in this field, is Myeong Soo Lee from the Korea Institute of Oriental Medicine, and he is also the founder of the International Society for Complementary Medicine Research and a consultant to the Cochrane Collaboration in complementary and alternative medicine. The second-ranked author was Edzard Ernst, a professor of complementary medicine at the University of Exeter, who has published over 30 articles together with Myeong Soo Lee, and their articles covered many diseases, such as musculoskeletal pain, stroke, cancer pain, arrhythmia, Parkinson's disease, rheumatoid arthritis, and others. ${ }^{19-24}$ Jianping Liu, Hugh MacPherson, Fanrong Liang, Ying Li, Zhishun Liu, Byung-Cheul Shin, and Tae-young Choi all formed a small cooperative group separately, whereas previously there was little cooperation among groups.

A total of 354 nodes and 445 links (Figure 4B) constituted the map of cited authors. J.P.T. Higgins was the most cited author, followed by D Moher, Hugh MacPherson, Edzard Ernst, and Klaus Linde. The top five cited authors in terms of centrality (Table 8) included G. Deng, Patricia M. Barnes, E. Manheimer, D.C. Cherkin, and M.K. Garcia. Higgins was the author of Cochrane Handbook for Systematic Reviews of Interventions Version 5.1.0, ${ }^{25}$ and it instructed researchers to follow scientific procedures to obtain credible results. D. Moher, ranking second, authored the PRISMA statement about systematic reviews and meta-analyses, ${ }^{26-28}$ and was always cited together with Higgins. Obviously, they have had significant academic influence in the field. It is worth mentioning that although the articles by the top-ranked cited authors were mostly published before 2010 , they have been frequently cited in the past 2 years, which suggests that acupuncture researchers are paying more attention to EBM. Only one article written by Klaus Linde about an RCT of acupuncture for migraine was a clinical study. ${ }^{29}$

\section{Analysis of Cited References}

A total of 173 references were extracted from 1189 records to analyze cited references (Figure 5A). The top 10 references in 


\section{A}

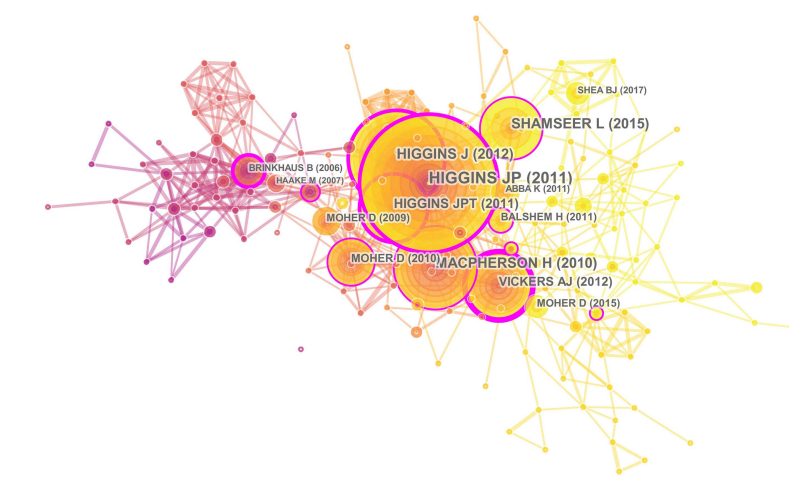

B

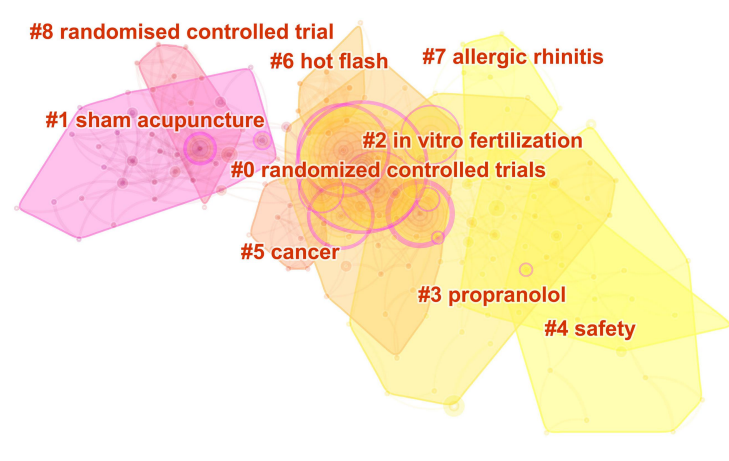

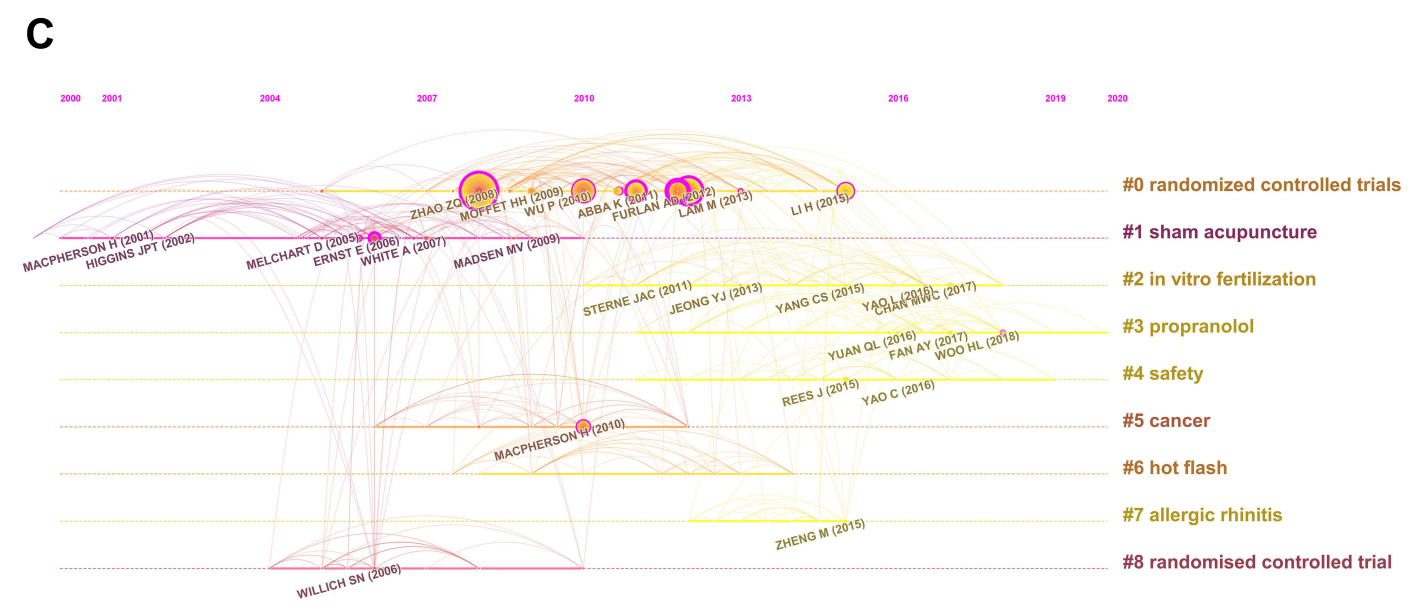

Figure 5 (A) shows the cited references map on acupuncture therapy related systematic review/meta analysis (The nodes in the map represent cited references, and lines between the nodes represent co-citation relationships. The various colors in the nodes represent the different years, and the larger the node area, the greater the number of citations. The purple ring represents centrality, and nodes with high centrality are considered as pivotal points in the literature.). (B) shows the cluster view map of cited references on acupuncture therapy related systematic review/meta analysis (The various colors in the clusters represent the different years, and the larger the cluster area, the greater the number of citations. The nodes in the map represent cited references. The purple ring represents centrality, and nodes with high centrality are considered as pivotal points in the literature.). (C) shows the timeline view map of cited references on acupuncture therapy related systematic review/meta analysis (The various colors of timeline and labels represent the different years. The nodes in the map represent cited references, and lines between the nodes represent co-citation relationships. And the larger the node area, the greater the number of citations. The purple ring represents centrality, and nodes with high centrality are considered as pivotal points in the literature.).

frequency and centrality are listed in Tables 9 and 10 . Furthermore, determine the knowledge structure of this field, cluster analysis was carried out. With a modularity Q of 0.6392 and a mean silhouette of 0.6803 , the cluster map (Figure 5B) consisted of 173 nodes and 538 links. And the value of modularity Q and mean silhouette meant that the clusters were rational.

According to the ranking of frequency and centrality in cited references, most were methodological studies, and they were always referred to as the standards for

Table 5 The Top 5 Frequency and Centrality of Countries on Acupuncture Therapy Related Systematic Review/Meta Analysis

\begin{tabular}{|l|l|l|l|l|c|}
\hline Rank & Freq & Countries & Rank & Centrality & Countries \\
\hline 1 & 683 & Peoples R China & 1 & 1.12 & Italy \\
2 & 196 & South Korea & 2 & 0.8 & Sweden \\
3 & 145 & England & 3 & 0.55 & Denmark \\
4 & 138 & USA & 4 & 0.41 & Switzerland \\
5 & 91 & Australia & 5 & 0.39 & Germany \\
\hline
\end{tabular}


Table 6 The Top 5 Frequency and Centrality of Institutions on Acupuncture Therapy Related Systematic Review/Meta Analysis

\begin{tabular}{|l|l|c|c|c|c|}
\hline Rank & Freq & Institutions & Rank & Centrality & Institutions \\
\hline 1 & 113 & Beijing Univ Chinese Med & 1 & 0.23 & Korea Inst Oriental Med \\
2 & 102 & Kyung Hee Univ & 2 & 0.18 & Beijing Univ Chinese Med \\
3 & 88 & Korea Inst Oriental Med & 3 & 0.15 & Kyung Hee Univ \\
4 & 87 & Chengdu Univ Tradit Chinese Med & 4 & 0.12 & Rmit Univ \\
5 & 71 & Guangzhou Univ Chinese Med & 5 & 0.11 & China Acad Chinese Med Sci \\
\hline
\end{tabular}

Table 7 The Top 5 Frequency and Centrality of Authors on Acupuncture Therapy Related Systematic Review/Meta Analysis

\begin{tabular}{|l|c|c|c|c|c|}
\hline Rank & Freq & Author & Rank & Centrality & Author \\
\hline 1 & 65 & Myeong Soo Lee & 1 & 0.15 & Myeong Soo Lee \\
2 & 48 & Edzard Ernst & 2 & 0.15 & Edzard Ernst \\
3 & 20 & Tae-young Choi & 3 & 0.06 & Hugh Macpherson \\
4 & 19 & Byung-Cheul Shin & 4 & 0.04 & Klaus Linde \\
5 & 19 & Zhishun LIU & 5 & 0.03 & Tae-young Choi \\
\hline
\end{tabular}

Table 8 The Top 5 Frequency and Centrality of Cited Authors on Acupuncture Therapy Related Systematic Review/Meta Analysis

\begin{tabular}{|l|l|c|c|c|c|}
\hline Rank & Freq & Cited Author & Rank & Centrality & Cited Author \\
\hline $\mathrm{I}$ & $49 \mathrm{I}$ & Higgins JPT & 1 & 0.38 & Deng G \\
2 & 294 & Moher D & 2 & 0.38 & Barnes Patricia M \\
3 & 234 & Macpherson H & 3 & 0.32 & Manheimer E \\
4 & 169 & Ernst E & 4 & 0.3 & Cherkin DC \\
5 & 107 & Linde K & 5 & 0.29 & Garcia MK \\
\hline
\end{tabular}

Table 9 The Top 10 Frequency of Cited References on Acupuncture Therapy Related Systematic Review/Meta Analysis

\begin{tabular}{|c|c|c|c|}
\hline Rank & Freq & Cited References & $\begin{array}{l}\text { Author and } \\
\text { Publication Year }\end{array}$ \\
\hline I & 190 & Cochrane Handbook for Systematic Reviews of Interventions: Cochrane Book Series ${ }^{25}$ & Higgins JP, 201I \\
\hline 2 & 100 & $\begin{array}{l}\text { Revised Standards for Reporting Interventions in Clinical Trials of Acupuncture (STRICTA): Extending } \\
\text { the CONSORT Statement }{ }^{30}\end{array}$ & Macpherson H, 2010 \\
\hline 3 & 92 & Convincing evidence from controlled and uncontrolled studies on the lipid-lowering effect of a statin & Higgins J, 2012 \\
\hline 4 & 91 & $\begin{array}{l}\text { Preferred reporting items for systematic review and meta-analysis protocols (PRISMA-P) 2015: } \\
\qquad \text { elaboration and explanation }{ }^{31}\end{array}$ & Shamseer L, 2015 \\
\hline 5 & 74 & The Cochrane Collaboration's tool for assessing risk of bias in randomised trials ${ }^{35}$ & Higgins JPT, 201 I \\
\hline 6 & 55 & Acupuncture for Chronic Pain Individual Patient Data Meta-analysis ${ }^{33}$ & Vickers AJ, 2012 \\
\hline 7 & 40 & Preferred reporting items for systematic reviews and meta-analyses: The PRISMA statement ${ }^{26}$ & Moher D, 2010 \\
\hline 8 & 30 & $\begin{array}{l}\text { Preferred reporting items for systematic review and meta-analysis protocols (PRISMA-P) } 2015 \\
\qquad \text { statement }^{27}\end{array}$ & Moher D, 2015 \\
\hline 9 & 27 & GRADE guidelines: 3 . Rating the quality of evidence ${ }^{32}$ & Balshem H, 20II \\
\hline 10 & 26 & Preferred Reporting Items for Systematic Reviews and Meta-Analyses: The PRISMA Statement ${ }^{28}$ & Moher D, 2009 \\
\hline
\end{tabular}


Table 10 The Top 10 Centrality of Cited References on Acupuncture Therapy Related Systematic Review/Meta Analysis

\begin{tabular}{|c|c|c|c|}
\hline Rank & Centrality & Cited References & $\begin{array}{l}\text { Author and } \\
\text { Publication Year }\end{array}$ \\
\hline I & 0.47 & Acupuncture for Chronic Pain Individual Patient Data Meta-analysis ${ }^{33}$ & Vickers AJ, 2012 \\
\hline 2 & 0.36 & Cochrane Handbook for Systematic Reviews of Interventions: Cochrane Book Series ${ }^{25}$ & Higgins JP, 20II \\
\hline 3 & 0.36 & The Cochrane Collaboration's tool for assessing risk of bias in randomised trials ${ }^{35}$ & Higgins JPT, 20II \\
\hline 4 & 0.3 & Acupuncture in patients with chronic low back pain: a randomized controlled trial & Brinkhaus B, 2006 \\
\hline 5 & 0.22 & $\begin{array}{l}\text { Convincing evidence from controlled and uncontrolled studies on the lipid-lowering effect of } \\
\qquad \text { a statin }\end{array}$ & HigginS J, 2012 \\
\hline 6 & 0.17 & $\begin{array}{l}\text { Revised STandards for Reporting Interventions in Clinical Trials of Acupuncture (STRICTA): } \\
\text { Extending the CONSORT Statement }{ }^{30}\end{array}$ & Macpherson $\mathrm{H}, 2010$ \\
\hline 7 & 0.16 & Preferred reporting items for systematic reviews and meta-analyses: The PRISMA statement ${ }^{26}$ & Moher D, 2010 \\
\hline 8 & 0.16 & Acupuncture for Chronic Pain: Update of an Individual Patient Data Meta-Analysis ${ }^{34}$ & Vickers AJ, 2018 \\
\hline 9 & 0.15 & $\begin{array}{l}\text { Preferred reporting items for systematic review and meta-analysis protocols (PRISMA-P) 2015: } \\
\qquad \text { elaboration and explanation }{ }^{31}\end{array}$ & Shamseer L, 2015 \\
\hline 10 & 0.15 & GRADE guidelines: 3 . Rating the quality of evidence ${ }^{32}$ & Balshem H, 20II \\
\hline
\end{tabular}

conducting research, which included the most cited reference, Cochrane Handbook for Systematic Reviews of Interventions and the second most-cited one, STRICTA. ${ }^{25,30}$ In addition, we found four PRISMA statements used to guide and standardize the plan for systematic review and meta-analysis. ${ }^{26-28,31}$ The Grading of Recommendations Assessment, Development and Evaluation (GRADE) guideline written by $\mathrm{H}$. Balshem was used to assess the quality of evidence, ${ }^{32}$ which is also listed in the two tables. In the ranking list, only one systematic review, written by A.J. Vickers in 2012 on acupuncture treatment of chronic pain ascended to the sixth place, ${ }^{33}$ and it was published in the Archives of Internal Medicine, which has been renamed JAMA Internal Medicine whose impact factor was 18.652. It was worth mentioning that this reference ranked first in centrality and his other meta-analysis of an individual patient suffering from chronic pain ranked eighth, ${ }^{34}$ which indicates the author and the two articles had a dominant influence in the field.

Then the cluster view map (Figure 5B) using loglikelihood tests (LLR) to find out the distribution from the keywords of literature comprised nine clusters where all Silhouettes were greater than 0.6, and through it we would have a general idea of popular research areas. The largest cluster was "randomized controlled trials," which contained 40 references. "Sham acupuncture," "in vitro fertilization," and "propranolol" were also active clusters. From the timeline view map (Figure 5C), clusters 2, 3, 4, and 7 were warm in color, which represents the latest research. Clusters 0 and 8 were similar, which meant they were relevant in some terms. Cluster 8 began in 2004 and lasted only 6 years, while cluster 0 began in 2005 and there were references cited with high frequency appearing almost every year after $2007 .^{25,30,31,33,35}$

\section{Analysis of Keywords}

The keywords co-occurrence map (Figure 6A), consisting of 487 nodes and 850 links, identified popular research topics, and the burst map (Figure 6B) reflected the research frontiers within a certain period. ${ }^{36}$ The top 10 keywords according to frequency and centrality are listed in Table 11. We found that "pain," "osteoarthritis," and "disability" were the main disorders in the meta-analysis of acupuncture therapy, which indicated that acupuncture has been extensively applied to the treatment of these diseases.

In addition to "osteoarthritis," the keywords "low back pain" and "knee" had a higher strength in the citation burst and lasted for about 3 years, indicating that meta-analysis research on these diseases had come to the forefront after 2010 but has been rapidly decreasing since 2014. As for interventions, "electroacupuncture" ranked in Table 11 indicating it gained in popularity, and we should select 


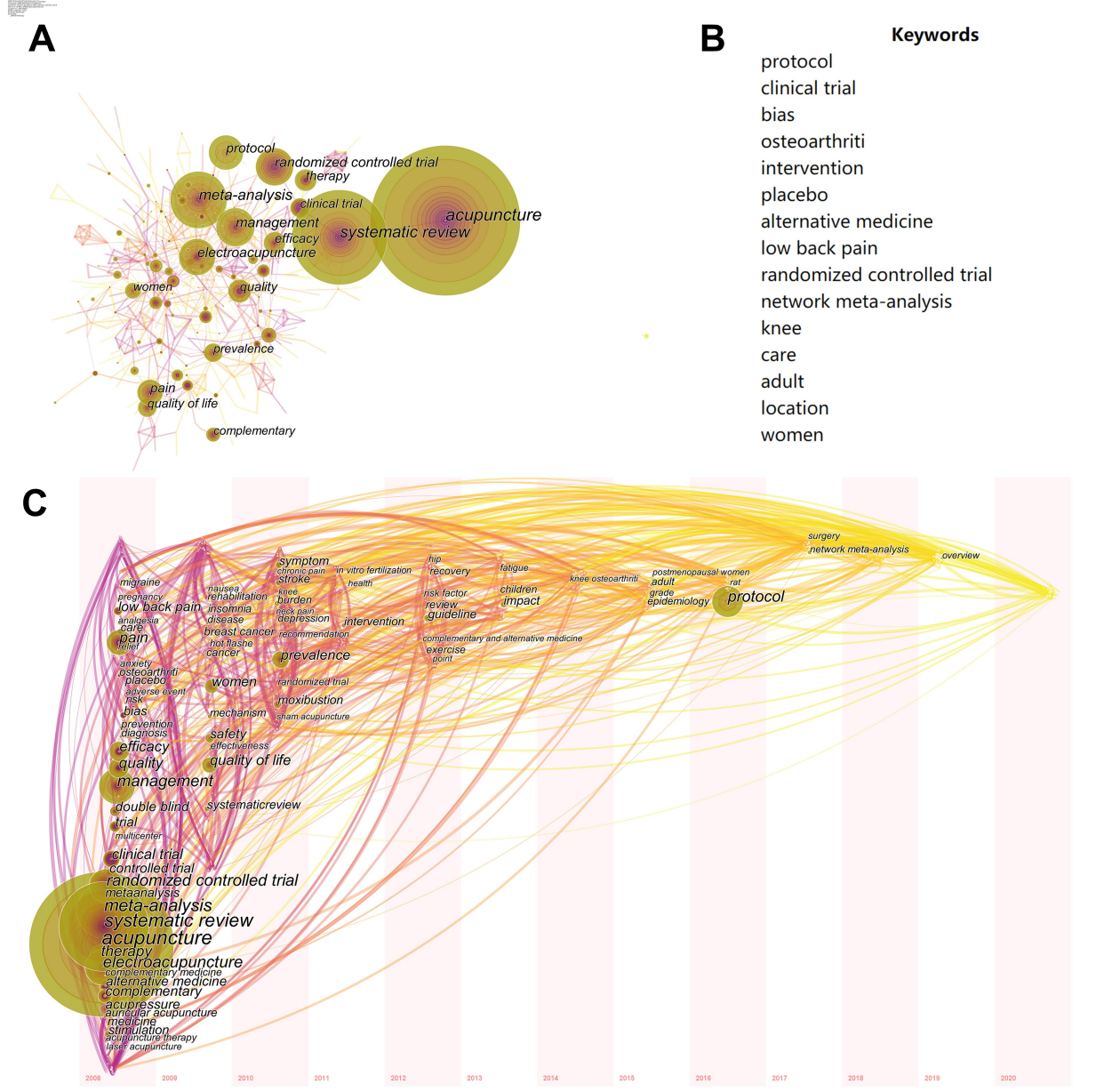

Figure 6 (A) shows the keyword map on acupuncture therapy related systematic review/meta analysis (The nodes in the map represent keywords. The lines between the nodes represent co-occurrence relationships. The various colors in the nodes represent the different years, and the larger the node area, the higher the frequency. The purple ring represents centrality, and nodes with high centrality are considered as pivotal points in the literature.). (B) shows the burst map of keywords on acupuncture therapy related systematic review/meta analysis (The red bars demonstrated that the keyword was cited frequently, the green bars showed that the keyword was cited infrequently.). (C) shows the timezone view map of keywords on acupuncture therapy related systematic review/meta analysis (The nodes in the map represent keywords. The lines between the nodes represent co-occurrence relationships. The various colors in the nodes represent the different years, and the larger the node area, the higher the frequency. The purple ring represents centrality, and nodes with high centrality are considered as pivotal points in the literature.).

the frequency based on the disease. Some studies discovered electroacupuncture could decrease pain significantly. ${ }^{37-40}$ After consulting the original literature, we also became aware of the links between it and some keywords such as "chronic spinal pain,"41 "pain relief,"42 and "analgesia". 43 "Randomized controlled trial" had a high frequency, because RCT had become more and more popular in clinical studies of TCM with the development of EBM. It is worth mentioning that double-blind ranked 11th. Blinded studies help avoid investigator bias and ensure the objectivity of the results. Because of the characteristics of acupuncture, it is hard to implement blinded studies. At present, the single-blind method was most widely used in participants or assessors only, and the double blind was set for both of them rather than the implementer. ${ }^{44-46}$

In the burst map (Figure 6B), the most recent burst keywords were "protocol," "network meta-analysis" and "adult." The keyword "placebo," with 7.8885 strength stood out from 2008 to 2012, showing that the selection and application of placebo had become a popular research topic in the clinical research on acupuncture during this period to some extent, and the commonly used placebo mainly included sham acupuncture, such as "shallow needling," "non-penetrating needles," and "non- acupuncture point". ${ }^{47}$ Participants were blinded by the above methods in some studies, but whether it was a genuine sham was still disputed and had not been widely used. ${ }^{48-50}$ 
Table II The Top 10 Frequency and Centrality of Keywords on Acupuncture Therapy Related Systematic Review/Meta Analysis

\begin{tabular}{|l|c|c|c|c|c|}
\hline Rank & Freq & Keyword & Rank & Centrality & Keyword \\
\hline 1 & 635 & acupuncture & 1 & 0.33 & acupressure \\
2 & 411 & systematic review & 2 & 0.25 & adverse event \\
3 & 249 & meta-analysis & 3 & 0.2 & exercise \\
4 & 175 & management & 4 & 0.17 & meta-analysis \\
5 & 168 & randomized controlled trial & 5 & 0.16 & intervention \\
6 & 167 & electroacupuncture & 6 & 0.14 & disability \\
7 & 148 & protocol & 8 & 0.13 & comparing acupuncture \\
8 & 124 & pain & 9 & 0.12 & osteoarthriti \\
9 & 112 & quality & 10 & 0.12 & complementary medicine \\
10 & 110 & therapy & & & acupuncture therapy \\
\hline
\end{tabular}

Then we applied a time-zone review to describe the trends and interactions of themes over time (Figure 6C). For interventions, most research was focused on "electroacupuncture," "acupressure," and "laser acupuncture," and more attention was given to "moxibustion" in 2010. After 2010, we found no subsequent studies of other acupuncture treatments. In terms of disease and therapy, "pain," "low back pain," "anxiety," and "osteoarthritis" were the research hot topics in 2008. In 2009, these were "insomnia," "cancer," "breast cancer," and "hot flash" and the application of therapy such as "rehabilitation" was expanded. In addition, research on "mechanism" began to increase. Later, research topics included on "stroke," "knee," "neck pain," "depression," "in vitro fertilization," "fatigue," "knee osteoarthritis," and "disorder," successively. In 2015, attention was paid to "epidemiology," and "rat" became a hot topic in the next year. It was interesting that the study populations in different periods were also different, and the subjects were mostly "women" in 2009, "children" in 2013, "postmenopausal women" in 2015, "men" in 2016, and "adult" in 2018. In terms of comparisons, "placebo" and "medicine" dominated in 2008, and "sham acupuncture" rose in 2010. Study types also changed. "Clinical trial," "RCT," "meta-analysis," and "systematic review" were popular in 2008 and some trial design factors such as "double blind" and "multicenter study" appeared used. No significant changes happened until 2012, when "guideline" became popular, as did "protocol" in 2016 and "network meta-analysis" in 2017. Finally, with respect to outcome indexes, "bias," "risk," and "quality" were the most common terms in 2008, and "safety," "quality of life," "effectiveness," "prevalence," and "recovery" were commonly observed in 2009 and thereafter. We predict that the research on hot topics in the future might concentrate on the design and improvement of acupuncture research protocols, as well as the development and promotion of guidelines for the application of acupuncture.

\section{Conclusion}

In summary, we investigated the scope of acupuncture therapy related to systematic reviews and meta-analysis research over the past 12 years through bibliometric analysis. The annual publications gradually increased and covered most disciplines, especially general medicine, neurology, obstetrics and gynecology, and oncology. Most publications appeared in the Journal of Alternative and Complementary Medicine. China and South Korea were the most prolific countries in publishing, and the most productive institution was Beijing University of Chinese Medicine, followed by KyungHee University. The author with the most publications was from South Korea as well, but the cooperation between different countries and institutions has been insufficient. Therefore, contacts and communication should be strengthened to promote the application of acupuncture therapy.

According to the cited references and keywords, the main disorder treated was pain, while EA gained the most attention in terms of interventions. Researchers attached great importance to international standards in the systematic review/meta-analysis of acupuncture therapy, such as the Cochrane Handbook, STRICTA, and PRISMA statements. It was difficult to implement the blinded studies and the selection of placebo was still not uniform. We predict that improving the quality of research may become a popular topic.

Finally, there are some limitations in this study. First, we analyzed only the articles and reviews in English recorded in the Web of Science Core Collection, so our findings may not be comprehensive enough. In addition, 
we manually deleted data irrelevant to the topic and some errors might have occurred in this process. However, we believe that our findings can still be used to describe the overall hot topics and trends in this field. It is necessary to further analyze the application of acupuncture, such as the specific application of types/modes of acupuncture and stimulation parameters, in order to understand the effectiveness of acupuncture. More rigorous research should be carried out in the future.

\section{Abbreviation}

EBM, evidence-based medicine; Freq, frequency; RCT, randomized controlled trial; EA, electro-acupuncture; STRICTA, Standards for Reporting Interventions in Clinical Trials of Acupuncture; PRISMA, preferred reporting items for systematic review and meta-analysis; GRADE, The Grading of Recommendations Assessment, Development and Evaluation.

\section{Acknowledgments}

The authors would like to express their appreciation to Professor CM Chen, who invented CiteSpace, which is free to use.

\section{Author Contributions}

All authors contributed to data analysis, drafting or revising the article, have agreed on the journal to which the article will be submitted, gave final approval of the version to be published, and agree to be accountable for all aspects of the work.

\section{Funding}

There is no funding to report.

\section{Disclosure}

The authors declare that there are no conflicts of interest regarding the publication of this paper.

\section{References}

1. Djulbegovic B, Guyatt GH. Progress in evidence-based medicine: a quarter century on. Lancet. 2017;390(10092):415-423. doi:10.1016/S0140-6736(16)31592-6

2. Horwitz RI, Hayes-Conroy A, Caricchio R, et al. From evidence-based medicine to medicine-based evidence. Am J Med. 2017;130 (11):1246-1250. doi:10.1016/j.amjmed.2017.06.012

3. McKenzie JE, Beller EM, Forbes AB. Introduction to systematic reviews and meta-analysis. Respirology. 2016;21(4):626-637. doi:10.1111/resp. 12783
4. Pandis N. The evidence pyramid and introduction to randomized controlled trials. Am J Orthod Dentofacial Orthop. 2011;140 (3):446-447. doi:10.1016/j.ajodo.2011.04.016

5. Murad MH, Asi N, Alsawas M, et al. New evidence pyramid. Evid Based Med. 2008;33(23):E887-E900. doi:10.1097/BRS.0b013e318186b276

6. Zhang JH, Li YP, Zhang BL. Evidence-based Chinese medicine: theory and practice. Zhongguo Zhong Yao Za Zhi. 2018;43(1):1-7. doi:10.19540/j.cnki.cjemm.20171127.001

7. Liu JP, Wang SC, Wu DR. Establishment and implementation of evidence-based clinical pathway of Chinese medicine. Zhongguo Zhong Xi Yi Jie He Za Zhi. 2011;31(1):115-119.

8. Yuan Q-L, Wang P, Liu L, et al. Acupuncture for musculoskeletal pain: a meta-analysis and meta-regression of sham-controlled randomized clinical trials. Sci Rep. 2016;6(1):30675. doi:10.1038/ srep30675

9. Chiu HY, Hsieh YJ, Tsai PS. Systematic review and meta-analysis of acupuncture to reduce cancer-related pain. Eur J Cancer Care. 2017;26:2. doi:10.1111/ecc.12457

10. Zhang R, Lao L, Ren K, et al. Mechanisms of acupuncture-electroacupuncture on persistent pain. Anesthesiology. 2014;120(2):482-503. doi:10.1097/ALN.0000000000000101

11. Paley CA, Johnson MI, Tashani OA, et al. Acupuncture for cancer pain in adults. Cochrane Database Syst Rev. 2015;2015(10): CD007753. doi:10.1002/14651858.CD007753.pub3

12. Cheng Y, Yuan Y, Jin Y, et al. Acupuncture for chronic pelvic inflammatory disease: a systematic review protocol. Medicine (Baltimore). 2018;97(13):e0225. doi:10.1097/MD.00000000 00010225

13. Lin SC, Cheifetz AS. The use of complementary and alternative medicine in patients with inflammatory bowel disease. Gastroenterol Hepatol. 2018;14(7):415-425.

14. Liang YD, Wang XY, Luo HL, et al. Metrology and visualization analysis of acupuncture and moxibustion related literature of Web of Science in the past 5 years by CiteSpace application. China J Trad Chin Med Pharm. 2017;32(05):2163-2168.

15. Chen C, SanJuan FI, Hou J. The structure and dynamics of cocitation clusters: a multiple-perspective cocitation analysis. J Am Soc Inf Sci Technol. 2010;61:7. doi:10.1002/asi.21309

16. Belter CW. Bibliometric indicators: opportunities and limits. J Med Libr Assoc. 2015;103(4):219-221. doi:10.3163/1536-5050.103.4.014

17. Pei W, Peng R, Gu Y, et al. Research trends of acupuncture therapy on insomnia in two decades (from 1999 to 2018): a bibliometric analysis. BMC Complement Altern Med. 2019;19(1):225. doi:10.1186/s12906-019-2606-5

18. Chen Y, Chen CM, Liu ZY, et al. Methodological functions of CiteSpace knowledge maps. Stud Sci Sci. 2015;33(02):242-253.

19. Lee MS, Pittler MH, Shin BC, et al. Bee venom acupuncture for musculoskeletal pain: a review. J Pain. 2008;9(4):289-297. doi:10.1016/j.jpain.2007.11.012

20. Kong JC, Lee MS, et al. Acupuncture for functional recovery after stroke: a systematic review of sham-controlled randomized clinical trials. Can Med Assoc J. 2010;182(16):1723-1729. doi:10.1503/ cmaj.091113

21. Choi TY, Lee MS, Kim TH, et al. Acupuncture for the treatment of cancer pain: a systematic review of randomised clinical trials. Support Care Cancer. 2012;20(6):1147-1158. doi:10.1007/s00.520012-1432-9

22. Lee S, Lee MS, Choi JY, et al. Acupuncture and heart rate variability: a systematic review. Auton Neurosci. 2010;155(1-2):5-13. doi:10.1016/j.autneu.2010.02.003

23. Lee MS, Shin BC, Kong JC, et al. Effectiveness of acupuncture for Parkinson's disease: a systematic review. Mov Disord. 2008;23 (11):1505-1515. doi:10.1002/mds.21993

24. Ernst E, Lee MS. Acupuncture for rheumatic conditions: an overview of systematic reviews. Rheumatology. 2010;49(10):1957-1961. doi:10.1093/rheumatology/keq180 
25. Higgins JP, Green S Cochrane handbook for systematic reviews of interventions version 5.1.0 (updated March 2011): the Cochrane Collaboration; 2011. www.handbook.cochrane.org. Accessed February 3, 2021.

26. Moher D, Liberati A, Tetzlaff J, et al. Preferred reporting items for systematic reviews and meta-analyses: the PRISMA statement. Int J Surg. 2010;8(5):336-341. doi:10.1016/j.ijsu.2010.02.007

27. Moher D, Shamseer L, Clarke M, et al. Preferred reporting items for systematic review and meta-analysis protocols (PRISMA-P) 2015 statement. Syst Rev. 2015;4(1):1. doi:10.1186/2046-4053-4-1

28. Moher D, Liberati A, Tetzlaff J, et al. Preferred reporting items for systematic reviews and meta-analyses: the PRISMA statement. BMJ. 2009;339:b2535. doi:10.1136/bmj.b2535

29. Linde K, Streng A, Jürgens S, et al. Acupuncture for patients with migraine: a randomized controlled trial. JAMA. 2005;293 (17):2118-2125. doi:10.1001/jama.293.17.2118

30. MacPherson H, Altman DG, et al. Revised Standards for Reporting Interventions in Clinical Trials of Acupuncture (STRICTA): extending the CONSORT statement. PLoS Med. 2010;7(6):e1000261. doi:10.1371/journal.pmed.1000261

31. Shamseer L, Moher D, Clarke M, et al. Preferred reporting items for systematic review and meta-analysis protocols (PRISMA-P) 2015: elaboration and explanation. BMJ. 2015;350:g7647. doi:10.1136/bmj. g7647

32. Balshem H, Helfand M, Schunemann HJ, et al. GRADE guidelines: 3. Rating the quality of evidence. J Clin Epidemiol. 2011;64(4):401406. doi:10.1016/j.jclinepi.2010.07.015

33. Vickers AJ, Cronin AM, Maschino AC, et al. Acupuncture for chronic pain: individual patient data meta-analysis. Arch Intern Med. 2012;172(19):1444-1453. doi:10.1001/archinternmed. 2012.3654

34. Vickers AJ, Vertosick EA, Lewith G, et al. Acupuncture for chronic pain: update of an individual patient data meta-analysis. J Pain. 2018;19(5):455-474. doi:10.1016/j.jpain.2017.11.005

35. Higgins JPT, Altman DG, Gøtzsche PC, et al. The Cochrane Collaboration's tool for assessing risk of bias in randomised trials. BMJ. 2011;343:d5928. doi:10.1136/bmj.d5928

36. Zheng K, Wang X. Publications on the association between cognitive function and pain from 2000 to 2018: a bibliometric analysis using CiteSpace. Med Sci Monit. 2019;25:8940-8951. doi:10.12659/ MSM.917742

37. Han JS. Induction of the release of central neuropeptides by peripheral electrical stimulation. J Peking Univ. 2002;(05):408-413.
38. Chen HJ, Li M, Chen L. Mechanism of acupuncture analgesia and endogenous cannabinoid system in integumentum commune. J Peking Univ. 2011;32(04):564-568.

39. Qu CL, Tang JS. Roles of ventrolateral orbital cortex in pain modulation and acupuncture analgesia. Sheng Li Ke Xue Jin Zhan. 2008;39(4):297-301.

40. Chen RW, Liu H, An JX, et al. Cognitive effects of electro-acupuncture and pregabalin in a trigeminal neuralgia rat model induced by cobra venom. J Pain Res. 2017;10:1887-1897. doi:10.2147/JPR.S140840

41. Yuan J, Purepong N, Kerr DP, et al. Effectiveness of acupuncture for low back pain: a systematic review. Spine. 2008;33(23):E887-900. doi:10.1097/BRS.0b013e318.186b276

42. Zhang CS, Yang AW, Zhang AL, et al. Sham control methods used in ear-acupuncture/ear-acupressure randomized controlled trials: a systematic review. J Altern Complement Med. 2014;20 (3):147-161. doi:10.1089/acm.2013.0238

43. Cho SH, Hwang EW. Acupuncture for primary dysmenorrhoea: a systematic review. BJOG. 2010;117(5):509-521. doi:10.1111/ j.1471-0528.2010.02489.x

44. Dyson-Hudson TA, Kadar P, LaFountaine M, et al. Acupuncture for chronic shoulder pain in persons with spinal cord injury: a small-scale clinical trial. Arch Phys Med Rehabil. 2007;88 (10):1276-1283. doi:10.1016/j.apmr.2007.06.014

45. Kim HM, Cho SY, Park SU, et al. Can acupuncture affect the circadian rhythm of blood pressure? A randomized, double-blind, controlled trial. J Altern Complement Med. 2012;18(10):918-923. doi:10.1089/acm.2011.0508

46. Yuan QL, Wang P, Liu L, et al. Acupuncture for musculoskeletal pain: a meta-analysis and meta-regression of sham-controlled randomized clinical trials. Sci Rep. 2016;6:30675. doi:10.1038/srep30675

47. Dincer F, Linde K. Sham interventions in randomized clinical trials of acupuncture: a review. Complement Ther Med. 2003;11 (4):235-242. doi:10.1016/s0965-2299(03)00124-9

48. Yong-Zhou W. Placebo effect of acupuncture: a critique and reflection on methodology of clinical research. Zhongguo Zhen Jiu. 2012;32(8):731-735.

49. Li JB. Research progress of placebo in clinical trial of acupuncture and moxibustion. J Clin Acupunct Moxibustion. 2009;25(01):53-54.

50. Moffet HH. Sham acupuncture may be as efficacious as true acupuncture: a systematic review of clinical trials. J Altern Complement Med. 2009;15(3):213-216. doi:10.1089/acm.2008.0356
Journal of Pain Research

\section{Publish your work in this journal}

The Journal of Pain Research is an international, peer reviewed, open access, online journal that welcomes laboratory and clinical findings in the fields of pain research and the prevention and management of pain. Original research, reviews, symposium reports, hypothesis formation and commentaries are all considered for publication. The manuscript

Submit your manuscript here: https://www.dovepress.com/journal-of-pain-research-journa management system is completely online and includes a very quick and fair peer-review system, which is all easy to use. Visit http:// www.dovepress.com/testimonials.php to read real quotes from published authors. 